\section{Papel del médico de familia en el diagnóstico concomitante de mieloma y amiloidosis primaria en una misma paciente. Caso clínico}

\author{
F. ANDREA RODRÍGUEZ-LAGOS ${ }^{1,2}$, JOSÉ V. SORLÍ İ,3,4,a, \\ MICHELLE C. CALVIÑO-NAVEIRA ${ }^{2,5}$, NURIA ESTAÑ CAPELL ${ }^{1}$
}

\section{Multiple myeloma associated with a secondary amyloidosis. Report of one case}

Multiple Myeloma is a myeloproliferative disorder of plasma cells, which may be complicated with secondary amyloidosis. We report a 48 year old woman consulting to primary care for weight loss and malaise. An initial laboratory study revealed a hypogammaglobulinemia with a monoclonal component and lambda light chains. These results motivated her derivation to hematology: her serum calcium was $11.8 \mathrm{mg} / \mathrm{dl}$, immunofluorescence showed a monoclonal component of lambda chains and urine Bence-Jones protein was positive. A bone marrow biopsy confirmed plasma cell infiltration. A Congo-red stain of a rectal biopsy was positive. The patient was treated with thalidomide, bortezomid and dexamethasone.

(Rev Med Chile 2018; 146: 1351-1355)

Key words: Amyloidosis; Congo Red; Multiple Myeloma; Plasma Cells.
${ }^{1}$ Hospital Universitario Dr. Peset, Servicio de Análisis Clínicos. Valencia, España.

${ }^{2}$ Grupo de trabajo (GdT) Sociedad

Valenciana y Española de Medicina

Familiar y Comunitaria (SoVaMFyC,

SEMFYC) de Genética Clínica y

Enfermedades Raras.

${ }^{3}$ Departamento Medicina Preventiva y

Salud Pública. Universitat de València y

CIBEROBN, España.

${ }^{4}$ Unidad mixta de investigación en

enfermedades raras FISABIO-UV.

${ }^{5}$ Centro de Salud de Vite. Santiago de

Compostela, A Coruña, España.

aphD.

Los autores declaran no tener conflictos de interés.

Trabajo no recibió financiamiento.

Recibido el 7 de junio de 2018, aceptado el 29 de octubre de 2018.

Correspondencia a:

Francy Andrea Rodríguez Lagos

Calle Melissa 69 Puerta 14, Paterna,

Valterna, Valencia

Código Postal: 46980, España.

Francyandreita@hotmail.com
E 1 mieloma múltiple (MM), se define como un desorden mieloproliferativo de células plasmáticas; casi siempre va precedido por un estado pre-maligno asintomático ${ }^{1}$. Se caracteriza por la proliferación clonal de células plasmáticas, que tienen capacidad de producir una proteína de carácter monoclonal, ésta se puede detectar en orina (Proteinuria de Bence-Jones) o suero en forma de una banda o componente monoclonal. Las células plasmáticas secretan inmunoglobulinas (Ig) de diversas clases (IgG, IgA, IgM, IgD e IgE $)^{2}$.

La incidencia MM en España se estima en 5 casos nuevos por $100.000 \mathrm{hab} / \mathrm{anno}^{2}$, incluye el $2 \%$ del total de cánceres, el 10\% de las neoplasias hematológicas y es la causa de mortalidad de $20 \%$ de las neoplasias hematológicas ${ }^{3}$.

La amiloidosis es una enfermedad de etiología desconocida, caracterizada por el depósito de una sustancia amiloidea en los espacios extracelulares de diversos órganos, condicionando alteraciones funcionales y estructurales. Esta sustancia, presenta un componente constante y un componente proteico variable que constituye la base para la clasificación patogénica de la amiloidosis. Existen tres tipos: el primero se denomina proteína AL y se presenta en la amiloidosis primaria (AL), siendo está la asociada a MM, el segundo contiene la llamada proteína AA y se presenta en pacientes con amiloidosis secundaria y el tercer tipo, se asocia con polineuropatía amiloidea familiar, es generalmente una molécula de amiloide transtiretina ${ }^{4}$. El diagnóstico de AL se basa en la histología del órgano afectado y la tinción de Rojo Congo positiva; la biopsia abdominal tiene sensibilidad de $57-85 \%$ y especificidad de $92-100 \%$ y la biopsia rectal sensibilidad de $84 \%{ }^{4}$.

La forma más común es la AL, la padecen alrededor de 75\% de los pacientes con Amiloidosis; 
Diagnóstico de mieloma y amiloidosis primaria en una misma paciente - F. A. Rodríguez-Lagos et al

5\% del total de los individuos afectados padecen amiloidosis secundaria, y menos de 5\% desarrollan una amiloidosis familiar. La incidencia anual de la AL es de 0,9 nuevos casos por 100.000 habitantes, con una media de edad de 65 años 5

Presentamos a una mujer que a través de la solicitud de un análisis de sangre por su médico de atención primaria (MAP) junto con la especificación y sospecha diagnóstica adecuada, lleva al equipo de laboratorio a realizar una extensión de las pruebas diagnósticas en busca de enfermedades relacionadas con el diagnóstico sugerido por su MAP; recibiendo todo el proceso de atención clínica, un diagnóstico precoz y un tratamiento oportuno y efectivo.

\section{Presentación del caso}

Mujer de 48 años que acude a su MAP por cuadro de 2 meses de evolución de pérdida de 9 $\mathrm{kg}$, astenia, hiporexia y naúseas; cuadro que inicia posterior al tratamiento con antiinflamatorios que tomaba por gonalgía izquierda; asociaba disnea de moderados esfuerzos, no ortopnea, no dolor torácico ni palpitaciones, sin tos, sin expectoración, ni hemoptisis. No otros síntomas sistemáticos asociados. Antecedentes de ser exfumadora desde hace 30 años, estudiada en Dermatología por sospecha de mastocitosis cutánea vs urticaria pigmentosa, leve elevación de triptasa sérica y ferropenia, sin otros antecedentes relevantes. A la exploración física presenta constantes vitales normales, regular estado general y apariencia caquéctica, palidez cutánea y discretos crepitantes en ambas bases pulmonares, el resto de la exploración sin ninguna alteración.

Su MAP solicita una analítica con bioquímica completa, hemograma y un proteinograma, plasmando en el volante de petición el diagnóstico de "Síndrome constitucional"; en el proteinograma hay una alteración en zona gamma no cuantificable, y ante el diagnóstico referido se amplían desde el hospital más pruebas, realizando una inmunofijación en gel de agarosa, con posterior informe y aviso vía telefónica a su MAP de la presencia de hipogammaglobulinemia y componente monoclonal de cadenas ligeras lambda, hipercalcemia $\mathrm{y}$ aumento discreto de transaminasas.

Posterior al aviso recibido del laboratorio, su MAP remite a la paciente a Hematología. La pa- ciente presenta empeoramiento de la disnea y el médico decide remitirla al servicio de urgencias; a su llegada no hay cambios en la exploración física, se decide ingreso en Medicina Interna y Hematología con sospecha inicial de Amiloidosis.

A su ingreso se realizan analíticas (Tabla 1), destacan las alteraciones de: Bilirrubina total: 3,36 mg/dl, Bilirrubina directa: 2,37 mg/dl, Bilirrubina indirecta: $0,99 \mathrm{mg} / \mathrm{dl}$, AST: $183 \mathrm{UI} / \mathrm{L}$, ALT: 165 UI/L, GGT: 623 UI/L, LDH: 304 UI/L, FA: $801 \mathrm{UI} / \mathrm{L}$; Calcio: 11,8 mg/d, Proteínas (orina $24 \mathrm{~h}$ ): 1,67 g/24 h; Microalbuminuria (orina24 h):73 mg/24 h, Inmunofluorescencia: componente monoclonal de cadenas ligeras lambda, Ratio CLL: 0,003, IgG: 322 mg/dl; IgA: 15 mg/ dl; IgM: $4 \mathrm{mg} / \mathrm{dl}$; Cadenas libres lambda suero: $2.450 \mathrm{mg} / \mathrm{l}$, Orina: Bence-Jones: positivo y componente cadenas ligeras lambda libres: $437 \mathrm{mg} / 24 \mathrm{~h}$, Serie ósea: lesiones líticas en ambos fémures y ramas isquio-púbicas. Durante su hospitalización realizan biopsia de médula ósea: infiltración 76\% por células plasmáticas y Rojo Congo negativo; inicialmente se realizan biopsias de glándula parótida y grasa subcutánea Rojo Congo negativas y finalmente ante la negatividad de las otras biopsias, se realiza biopsia de recto Rojo Congo positivo, Resonancia Magnética cardíaca sugestiva de patología infiltrativa. Inician tratamiento con bortezomid, dexametasona y talidomida por 4 ciclos.

\section{Discusión y Comentarios}

Debemos destacar el papel del MAP en la realización de diagnósticos tempranos y derivaciones oportunas a otros servicios especializados, de enfermedades consideradas como raras y que tienen alta morbilidad; al igual que insistir en la importancia de la comunicación del laboratorio y el MAP, ya que los facultativos que sospechan una entidad patológica pueden recibir una orientación clínica, realizando conjuntamente y con los resultados analíticos diagnósticos de patologías complejas. Por otra parte, los facultativos del laboratorio realizan avisos oportunos al médico solicitante, consiguiendo de esta forma beneficios para el paciente, resultando fundamental para la asistencia y coordinación del sistema sanitario, el papel de complementariedad entre atención primaria con niveles más complejos a nivel hospitalario y de laboratorio. 


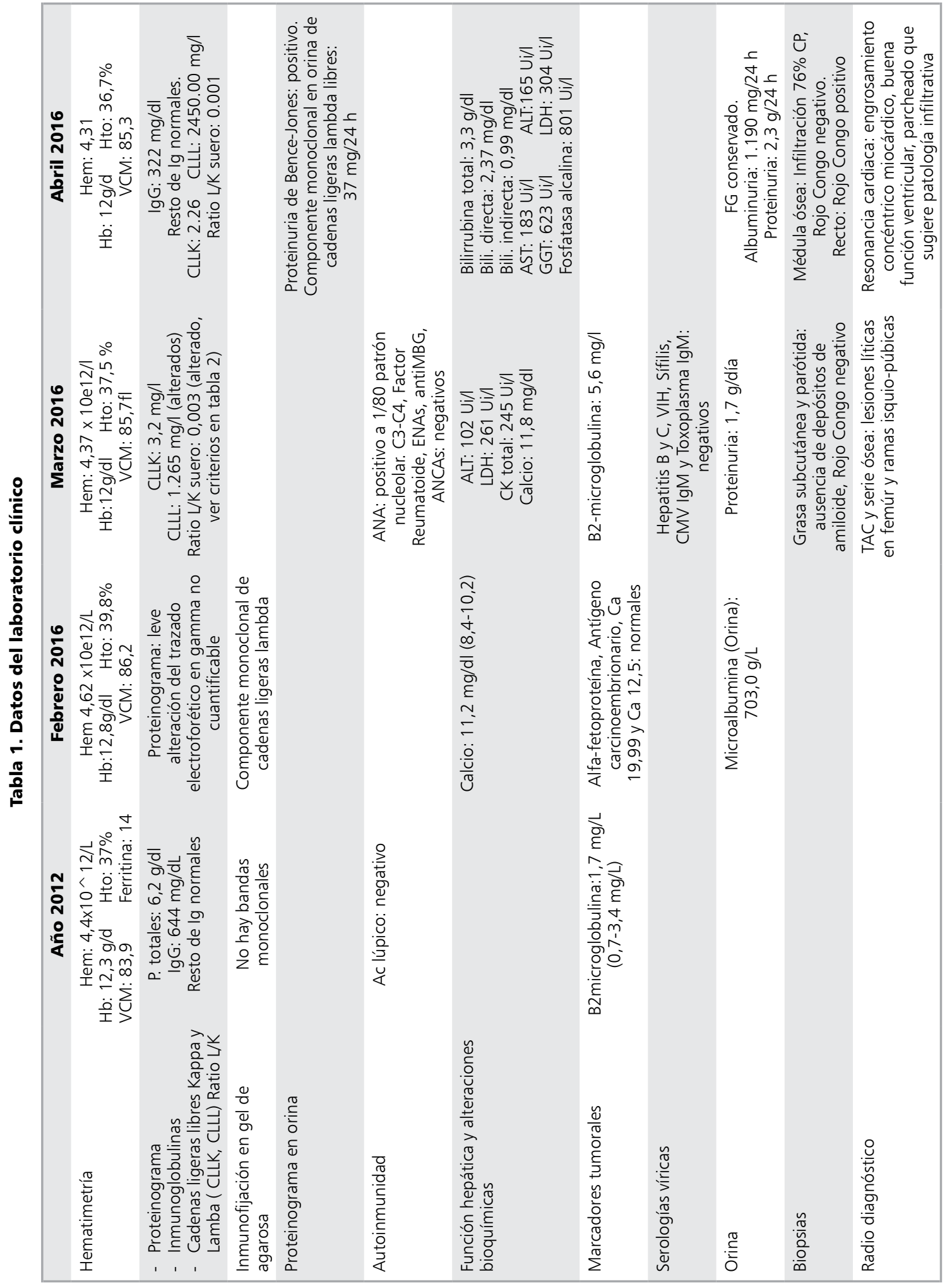


Diagnóstico de mieloma y amiloidosis primaria en una misma paciente - F. A. Rodríguez-Lagos et al

Tabla 2. Criterios diagnósticos para gammapatias monoclonales (International Myeloma Working Group, 2014)

\begin{tabular}{|c|c|c|c|c|c|c|c|c|}
\hline $\begin{array}{l}\text { Tipo de } \\
\text { gamapatía } \\
\text { monoclonal }\end{array}$ & $\begin{array}{c}\text { GMSI } \\
\text { no } \\
\text { IgM }\end{array}$ & $\begin{array}{l}\text { GMSI } \\
\text { IgM }\end{array}$ & $\begin{array}{c}\text { GMSI } \\
\text { por } \\
\text { CL }\end{array}$ & SMM & \multicolumn{2}{|c|}{$\begin{array}{l}\text { Mieloma } \\
\text { Múltiple* }\end{array}$} & $\begin{array}{c}\text { MM } \\
\text { oligosecretor }\end{array}$ & $\begin{array}{l}\text { Macroglobulinemia } \\
\text { de Waldeström }\end{array}$ \\
\hline CRAB O amiloidosis & No & No & No & No & No & Sí & Sí & $\begin{array}{l}\text { Síndrome de } \\
\text { hiperviscocidad, } \\
\text { Neuropatía } \\
\text { Crioglobulinemía }\end{array}$ \\
\hline $\begin{array}{l}\text { Células plasmáticas } \\
\text { en } \mathrm{MO}(\%)\end{array}$ & $<10$ & $<10$ & $<10$ & 10 a 60 & $>60 * *$ & $>10$ & $\geq 10$ & $\geq 10$ \\
\hline $\begin{array}{l}\text { Componente } \\
\text { monoclonal en } \\
\text { suero }(\mathrm{g} / \mathrm{L})\end{array}$ & $<30$ & $<30$ & & & \multirow{2}{*}{\multicolumn{2}{|c|}{$\begin{aligned}> & 30 \\
& 0 \\
> & 500\end{aligned}$}} & No detectable & \\
\hline $\begin{array}{l}\text { Componente } \\
\text { monoclonal orina } \\
\text { (mg/24h) }\end{array}$ & & & $<500$ & & & & & \\
\hline $\begin{array}{l}\text { Cociente } \kappa / \lambda \text { libres } \\
\text { en suero }\end{array}$ & & & $\begin{array}{c}<0,26 \\
0< \\
1,65\end{array}$ & & \multirow{3}{*}{$\begin{array}{c}(>100 \\
y \\
\left.>100^{*}\right)\end{array}$} & & $\begin{array}{l}<0,260 \\
<1,65\end{array}$ & \\
\hline $\begin{array}{l}\text { Cadena ligera } \\
\text { libre en suero } \\
\text { involucrada }\end{array}$ & & & & & & & & \\
\hline $\begin{array}{l}\text { Cadena pesada IFs } \\
\text { involucrada (mg/L) }\end{array}$ & & & Negativa & & & & & \\
\hline $\begin{array}{l}\text { Lesión vista en } \\
\text { RNM }\end{array}$ & & & & & \multicolumn{2}{|l|}{$\geq 1 * *$} & & \\
\hline $\begin{array}{l}\text { \% de progresión } \\
\text { anual }\end{array}$ & 1 & 1,5 & 0,3 & & & & & \\
\hline
\end{tabular}

CRAB: Hipercalcemia, enfermedad renal, anemia, lesión ósea. IFs: inmunofijación. GMSI: gammapatía monoclonal de significado incierto. SMM: mieloma múltiple asintomático. *Nueva definición de MM: > 10\% de CP en MO o plasmocitoma óseo o extramedular y además CRAB o $\geq 1$ de los factores marcados como**. Manual de Formación Continuada 2015-2016. La efectividad en la Medicina del Laboratorio Clínico. AEBM.

La AL cursa con pérdida de peso, astenia y una tríada clínica (insuficiencia cardiaca, proteinuria sin diabetes y neuropatía periférica) que debe despertar la sospecha clínica ${ }^{5,6}$, asociado puede tener un componente monoclonal sérico y/o urinario o una población monoclonal de células plasmáticas en la médula ósea ${ }^{6}$; destacando que el MM también puede presentar los mismos hallazgos analíticos, pero, con síntomas asociados a MM, como anemia, dolores óseos, lesiones osteolíticas y plasmocitomas. La literatura describe que $30 \%$ de pacientes con MM tienen depósitos de materiales amiloideos asintomáticos, y entre $10-15 \%$ de estos pacientes van a desarrollar AL sintomática ${ }^{2,6}$.

A favor del diagnóstico de AL encontramos clínicamente la disnea, astenia y pérdida de peso, además una imagen sugestiva de infiltración cardiaca amiloidea en la Resonancia, alteración en la función hepática y contradictoriamente biopsias iniciales Rojo Congo negativas, cabe destacar que la AL puede cursar con una proporción elevada de células plasmáticas en médula ósea y elevada proteinuria de cadenas ligeras ${ }^{3}$, como lo tiene nuestra paciente; pero ante la clínica inicial inespecífica, las biopsias iniciales negativas que descartaban AL y debido el gran número de criterios positivos para MM (criterios diagnósticos International Myeloma Working Group, 2014) (Tabla 2) ${ }^{1,2,5}$, como son células plasmáticas en médula ósea $>10 \%$ (> 60\%), hipercalcemia, lesiones líticas (dos 
síntomas CRAB), CLL > $100 \mathrm{mg} / \mathrm{L}$, componente monoclonal en suero cadenas ligeras y en orina de cadenas ligeras libres (Bence-Jones), se consideró para fines terapéuticos como MM, obteniendo buena respuesta clínica; posteriormente ante la positividad de Rojo Congo en la biopsia de recto, se asocia el diagnóstico de AL y realizan trasplante de médula ósea con mejoría extraordinaria.

A pesar de la dificultad diagnostica, su MAP realizó un adecuado proceso diagnóstico; de forma hospitalaria se efectuaron las pruebas pertinentes y se instauró el tratamiento adecuado, contribuyendo de esta forma a una mejoría de la paciente.

\section{Referencias}

1. Rajkumar SV, Dimopoulos MA, Palumbo A, Blade J, Merlini G, Mateos MV, et al. International Myeloma Working Group updated criteria for the diagnosis of multiple mieloma. Lancet Oncol 2014; 15 (12): e538-48.

2. Resumen de Oncoguia Mieloma Múltiple 2014. Versión
2.1. Grupo Cooperativo para el Estudio de Gammapatías Monoclonales de Castilla y León. 2014. Disponible en : http://www.sehh.es/es/documentos/guias-y-documentos/2839-oncoguia-mieloma-multiple-2014.html

3. Cibeira MT, Bladé J. Amiloidosis primaria: diagnóstico, pronóstico y tratamiento. Haematologica/edición española 2011; 96 (Extra 1): 13-8.

4. Kamal WS, Taib T, Noor NM, Jong TJ. Indian. Myeloma Associated Primary Systemic Amyloidosis. J Dermatol 2016; 61 (1): 124.

5. Sáez-Benito Godino A, Rodríguez Bayona B, Hernández Pacho F, Rico Rodríguez M. Gammapatías monoclonales: nuevos consensos. Qué aportan las cadenas ligeras libres y las parejas cadena pesada-cadena ligera. En Manual de Formación Continuada 2015-2016, La efectividad en la Medicina del Laboratorio Clínico, España. Editor: Asociación Española de Biopatología Médica (AEBM) 2015; p. 29-47.

6. Goldschmidt H, Lannert H, Bommer J, Ho AD. Multiple myeloma and renal failure. Nephrol Dial Transplant 2000; 15 (3) :301-4. 